\title{
Análise e modelagem da evolução temporal da posse de autos na Região Metropolitana de São Paulo
}

\author{
Letícia Marconcim Pfeiffer e Orlando Strambi \\ Escola Politécnica da Universidade de São Paulo, Brasil
}

RESUMO: O objetivo deste trabalho é estudar o comportamento da posse de autos familiar na Região Metropolitana de São Paulo, buscando analisar a evolução ocorrida entre 1987 e 1997. Foi utilizado um modelo logit multinomial que considera variáveis sociodemográficas e econômicas que influenciam o comportamento das famílias: tamanho da família, número de trabalhadores, número de estudantes, presença ou não de crianças, sexo do chefe, idade do chefe e a renda familiar. Os resultados apontaram mudanças de comportamento ao longo do tempo com relação às variáveis envolvidas, verificando-se uma redução da importância dessas características familiares para explicar a posse de autos, destacando-se o efeito decrescente da renda ao longo do tempo. Esses resultados sugerem que vem crescendo a importância de outros fatores na decisão de posse de auto das famílias, provavelmente relacionados com o mercado de veículos e com políticas urbanas e de transportes.

ABSTRACT: The objective of this work is to study family auto ownership behavior in the Metropolitan Area of São Paulo, by looking at its evolution during the period between 1987 and 1997. The analysis was based on the results of a multinomial logit model considering socio-demographic and economic attributes influencing family auto ownership: family size, number of workers, number of students, presence of children in the family, age and sex of the family head and family income. Results indicate significant behavioral changes with respect to the analysis variables during the period considered. In general, their importance as explanatory variables decreases from 1987 to 1997, including a decline in the influence of income on auto ownership behavior. These results suggest the growing importance of other factors on the auto ownership decision of families, probably related to car market characteristics and urban and transportation policies.

\section{INTRODUÇÃO}

O crescimento da motorização na Região Metropolitana de São Paulo - RMSP tornou-se intenso nas últimas décadas. As informações provenientes das pesquisas Origem-Destino realizadas indicam que o aumento no número de automóveis particulares foi significativo entre 1987 e 1997 , enquanto o índice de mobilidade, medido através do número médio de viagens motorizadas da população, apresenta uma intrigante diminuição. Estes resultados confirmam em geral o comportamento que tem sido relatado na literatura sobre as cidades dos países em desenvolvimento, contrariamente ao observado em cidades de países desenvolvidos onde o crescimento da motorização está diminuindo, principalmente pela proximidade de níveis esperados de saturação (Dargay e Gately, 1999; Kitamura e Kostyniuk, 1986).

O crescimento sistemático observado se deve provavelmente a mudanças no comportamento e nos hábitos das famílias e indivíduos com relação à posse de autos. Também são importantes as alterações na composição da população com relação a características sociodemográficas e econômicas como tamanho da família, renda, número de trabalhadores, presença de crianças etc., além de fatores externos, como a queda da qualidade do transporte coletivo, crescimento da competitividade do mercado automobilístico (facilidade de aquisição de automóveis), mudanças nos padrões de uso do solo entre outros. A posse de autos é um fator importante na determinação das decisões de famílias e indivíduos com relação às suas viagens e aos modos de transporte que utilizam. O objetivo deste trabalho é analisar o comportamento da posse de autos familiar na RMSP, em particular sua evolução no período entre 1987 e 1997, com relação às características das famílias.

\section{REVISÃO BIBLIOGRÁFICA}

Uma classificação comumente encontrada na literatura sobre os modelos de posse de autos é baseada no nível de agregação dos dados empregados no desenvolvimento e aplicação dos modelos. As estimativas e previsões da posse de autos podem ser obtidas através de modelos agregados, que fazem a estimativa em nível zonal e/ou regional, ou através de modelos desagregados, que usam a família ou o indivíduo como unidade de decisão, sendo as previsões zonais e regionais obtidas pela agregação das famílias (ou indivíduos). Atualmente outros tipos de 
modelos são utilizados, como os modelos de análise longitudinal, que analisam a evolução do comportamento familiar e/ou individual ao longo do tempo com relação à posse de autos, geralmente usando dados desagregados.

Os modelos de escolha discreta de posse de autos são modelos desagregados usados para analisar situações na qual a família faz uma escolha entre um conjunto (finito e exaustivo) de alternativas de posse de autos (mutuamente exclusivas). Estes modelos são atualmente os mais empregados na modelagem desagregada da posse de autos.

Diferentes hipóteses sobre o comportamento podem ser representadas através dos modelos de escolha discreta. O mecanismo mais empregado é o de resposta não ordenada, cuja hipótese de comportamento baseia-se no princípio da maximização da utilidade proveniente da teoria do consumidor, ou seja, perante um conjunto de alternativas, será escolhida a alternativa que produzir para a família a maior utilidade. Um segundo mecanismo empregado nos modelos de escolha discreta é o de resposta ordenada, baseado na hipótese de que um índice de propensão latente contínuo determina o nível de posse de autos. Neste tipo de mecanismo, o nível de posse de autos corresponde a uma divisão ordenada da linha real. A propensão latente da determinação da posse de autos familiar é especificada como sendo a soma de um componente determinístico e de um componente aleatório, sendo o último representado por uma função de densidade de probabilidade contínua.

Bhat e Pulugurta (1998) avaliaram os dois mecanismos comportamentais descritos acima para tentar identificar qual deles representa melhor o processo de decisão da posse de autos no âmbito familiar. Foram examinadas as estruturas teóricas por trás dos mecanismos de resposta ordenada e de resposta não ordenada, além dos desempenhos empíricos quando utilizados no desenvolvimento de modelos de posse de autos. Foram comparados os modelos logit ordenado e logit multinomial, estimados para vários conjuntos de dados. Utilizou-se nas especificações dos modelos diversas variáveis socioeconômicas e variáveis que capturam os atributos do ambiente de atividades e de viagens da família, como a localização da residência.

Considerando as diferentes estruturas dos modelos analisados, os resultados obtidos e os testes estatísticos realizados, os autores concluíram que ambos os modelos fornecem estimativas razoáveis. Entretanto, o modelo logit multinomial é capaz de capturar um padrão flexível dos efeitos de elasticidade das variáveis entre as alternativas, o que já não acontece com o modelo logit ordenado, que apresenta uma tendência mais rígida com relação aos efeitos de elasticidade. As diferenças nos efeitos de elasti- cidade mostram que as previsões com os modelos logit ordenado e logit multinomial são bem distintas em resposta às mudanças temporais das características socioeconômicas familiares e características do ambiente de atividades e viagens consideradas nas especificações.

A avaliação do desempenho dos modelos através de medidas de ajuste na amostra de estimação e na amostra de validação, assim como os resultados obtidos pela aplicação do modelo logit ordenado e do modelo logit multinomial em vários conjuntos de dados, mostraram que o modelo logit multinomial superou o modelo logit ordenado em todas as situações, levando os autores a concluir que a utilização do modelo logit ordenado na modelagem da posse de autos conduz a previsões incorretas e imprecisas. Sob o ponto de vista comportamental, os resultados indicam que o mecanismo de escolha de resposta não ordenada é o que melhor representa o processo da decisão familiar com relação à posse de autos.

Modelos de posse de autos utilizando formulações logit e probit não ordenados foram desenvolvidos por diversos pesquisadores como Purvis (1996), Ryan e Han (1999), Palma e Rochat (2000) e Chu (2002). Entretanto, o que pode ser observado é que a formulação mais empregada no desenvolvimento de modelos de posse de autos é a logit multinomial, devido à facilidade de estimação dos parâmetros presentes no modelo e simplicidade com relação à sua estrutura, quando comparados com formulações mais elaboradas, como os modelos logit hierárquico e probit multinomial.

\section{METODOLOGIA}

A metodologia utilizada no trabalho permite identificar as principais características familiares que afetam as decisões relacionadas com a posse do automóvel, assim como capturar as mudanças de seus efeitos ao longo do tempo. Foram utilizados os dados das pesquisas Origem-Destino - OD realizadas pela Companhia do Metropolitano de São Paulo - METRÔ para os anos de 1987 e 1997 na Região Metropolitana de São Paulo - RMSP. Após o tratamento dos dados, foram obtidas amostras de 24.358 e de 17.038 registros de famílias (unidade de análise escolhida) para os anos de 1987 e 1997, respectivamente, onde se buscou uma padronização das informações nos dois anos de pesquisa (Pfeiffer, 2004).

A metodologia proposta considerou três etapas distintas:

- a primeira etapa teve como objetivo principal estudar, ainda que de forma preliminar e exploratória, os efeitos de variáveis sociodemográficas 
e econômicas selecionadas sobre a posse de autos, visando auxiliar a especificação do modelo de posse de autos. Esta etapa considerou apenas os dados da amostra de famílias de 1987, para controlar possíveis efeitos de variação no tempo. Foram feitas análises dos gráficos do nível médio da posse de autos em função das variáveis selecionadas, como também se aplicou uma técnica de modelagem de segmentação - CHAID - que permite identificar interações relevantes entre variáveis;

- na segunda etapa foi estimado um modelo logit multinomial de posse de autos para a amostra de famílias de 1987, com o objetivo de buscar uma especificação adequada, que pudesse posteriormente ser estendida para incorporar os efeitos temporais;

- na terceira etapa foi estimado um modelo logit multinomial a partir da amostra conjunta de famílias de 1987 e 1997. Estendeu-se a especificação do modelo-base de 1987, com a inclusão de termos que capturassem a alteração ao longo do tempo dos efeitos das diferentes variáveis sobre a posse de autos familiar.

O CHAID é uma técnica estatística para análise de segmentação baseada em uma variável resposta (Kass, 1980). Este tipo de modelo define segmentos homogêneos de uma população com relação a uma variável resposta (ou dependente). Através deste método, categorias (também chamadas valores ou níveis) das variáveis independentes que são homogêneas com relação ao critério de segmentação (a variável resposta) são agrupadas, enquanto que categorias heterogêneas são mantidas separadas (Magidson, 1994). A técnica permite identificar os efeitos conjuntos das variáveis explicativas sobre a variável dependente, assim como a categorização mais adequada para essas variáveis e interações importantes entre categorias (Strambi e van de Bilt, 1998).

\subsection{Etapa 1}

O primeiro passo dessa etapa foi a seleção das variáveis a serem consideradas. Diversas variáveis sócioeconômicas e demográficas correlacionam-se com a posse de automóveis, no âmbito familiar e individual. Variáveis como renda, número de trabalhadores, tamanho da família, número de estudantes e o número de crianças são as mais utilizadas em modelos de posse de autos desagregados (Chu, 2002; Dargay e Vythoulkas, 1999; Dargay, 2001; Jager e Scheltes, 1985; Karlaftis e Golias, 2002; Prevedouros e Schofer, 1992; Purvis, 1996; Ryan e Han, 1999). Os atributos que caracterizam a estrutura familiar selecionados para este estudo foram: tamanho da família, número de trabalhadores, número de estudan- tes, presença ou não de crianças na família, sexo do chefe da família, idade do chefe da família e renda familiar.

A variável dependente, número de automóveis, foi representada por uma variável categórica que classifica as famílias em 4 categorias: $0,1,2$ e 3 ou mais automóveis; a variável tamanho da família foi segmentada em 8 categorias do número de pessoas: $1,2,3,4,5,6,7$ e 8 ou mais; o número de trabalhadores, por sua vez está sendo representado por 6 categorias: $0,1,2,3,4,5$ ou mais trabalhadores; a variável número de estudantes apresenta 4 categorias: $0,1,2,3$ ou mais estudantes. A variável renda familiar é apresentada em múltiplos de salários mínimos; para a realização das análises no CHAID foi dividida em 8 categorias. Diversas formas funcionais foram consideradas na estimação do modelo para representar o efeito da renda sobre a posse de autos familiar.

Para interpretar os efeitos causados pelas crianças com relação à posse de automóveis, adotou-se uma variável dummy, que identifica a presença ou não de crianças na família; esta variável assume valor 0 ou 1 , sendo que 1 indica a presença de um atributo e 0 a ausência deste atributo. O sexo do chefe da família também está sendo representado por uma variável dummy, que assume valor 0 quando o chefe é do sexo masculino, e assume valor 1 quando o chefe é do sexo feminino. A idade do chefe foi tratada como uma variável contínua para estimação dos modelos; para eliminar problemas devido a diferenças de escala entre as variáveis, foi dividida por 10 . Nas análises exploratórias com o CHAID, a idade do chefe foi dividida em 7 categorias.

\subsubsection{Resultados das análises gráficas e do CHAID com a amostra de famílias de 1987}

Foram elaborados gráficos relacionando o nível médio de posse de autos familiar com cada uma das variáveis explicativas selecionadas. Sua análise possibilitou a determinação de representações mais adequadas para as variáveis, assim como a observância de alguns aspectos referentes aos seus comportamentos, descritos a seguir:

- as variáveis tamanho da família e idade do chefe apresentam um efeito não linear sobre a posse de autos, que cresce inicialmente, para depois diminuir. Embora esse efeito seja esperado para a idade do chefe (ver, por exemplo, Strambi et al., 2000), ele surpreende para o tamanho da família. Tal resultado levou à inclusão de um termo quadrático para essas variáveis na especificação do modelo, para capturar este efeito;

- as variáveis número de trabalhadores e número de estudantes estão associadas a um crescimento 
do nível médio da posse de autos à medida que aumenta o número de pessoas que trabalham ou estudam na família;

- a análise da variável presença ou não de crianças indica comportamentos distintos entre famílias sem crianças e famílias com crianças quando se trata da posse de autos familiar: a maior concentração de crianças encontra-se nas famílias sem automóveis, para os demais níveis de posse de autos as diferenças continuam, indicando que famílias com crianças apresentam níveis mais baixos de posse de autos;

- com relação ao sexo do chefe verifica-se que famílias com chefes mulheres têm níveis de posse de autos menores se comparados com famílias chefiadas por homens.

Essas análises mostram apenas a associação entre o nível médio de posse de autos e uma variável específica, sem controlar o efeito das demais. Considerando que algumas das variáveis selecionadas apresentam elevada correlação entre si, realizou-se uma análise adicional usando o CHAID.

Quando se realizou a análise com o CHAID, a renda familiar foi a primeira variável a segmentar a amostra. A variável selecionada para prosseguir a segmentação foi distinta, dependendo da faixa de renda: para os níveis mais baixos de renda, o sexo do chefe; para as faixas de renda intermediárias, o número de trabalhadores; e para as faixas de renda mais altas, a idade do chefe. A maioria dos comportamentos observados pelos gráficos foi confirmada pelo CHAID. Verificou-se, porém, que para uma dada categoria (ou categorias agregadas) de renda, o efeito do número de trabalhadores muda com relação ao observado para a média da amostra de famílias em 1987, tornando-se negativo com o aumento do número de trabalhadores. Nas famílias onde há um maior número de trabalhadores, estes apresentam uma menor contribuição individual para a renda familiar, caracterizando padrões de consumo que reduzem a probabilidade de posse de autos.

\subsection{Etapa 2}

A partir dos resultados obtidos na primeira etapa, foram especificadas as funções de utilidade para o modelo logit multinomial de posse de autos familiar. O modelo foi estimado usando apenas a amostra de 1987, por simplicidade e por esta conter melhores informações sobre a renda familiar; um dos principais objetivos desta etapa foi encontrar uma representação adequada da renda nas funções de utilidade. O software LIMDEP (Greene, 1998) foi utilizado na estimação dos modelos, com base no princípio da máxima verossimilhança.
A formulação geral para a expressão de utilidade de cada alternativa da posse de autos é representada pela equação a seguir:

$$
\mathrm{U}_{\mathrm{jf}}=\theta_{\mathrm{j} 1}+\sum_{\mathrm{i}} \theta_{87 \mathrm{ij}} \cdot \mathrm{VAR}_{\mathrm{if}}+\varepsilon_{\mathrm{jf}}
$$

em que:

$$
\begin{aligned}
& \mathrm{U}_{\mathrm{jf}}: \text { Utilidade associada pela família } \mathrm{f} \text { à al- } \\
& \text { ternativa } \mathrm{j} \text { (número de automóveis: } 0,1,2 \text {, } \\
& 3 \text { ou mais); } \\
& \theta_{\mathrm{j} 1}: \text { Constante específica da alternativa } \mathrm{j} \text {; } \\
& \theta_{87 \mathrm{j}} \text { : Coeficiente da variável } \mathrm{i} \text {, específico } \\
& \text { para a alternativa j, correspondente à amos- } \\
& \text { tra de famílias de 1987; } \\
& \mathrm{VAR}_{\mathrm{if}} \text { : Tamanho da família, (tamanho da } \\
& \text { família) }{ }^{2} \text {, número de trabalhadores, número } \\
& \text { de estudantes, presença ou não de crianças, } \\
& \text { sexo do chefe, idade do chefe, (idade do } \\
& \text { chefe) }{ }^{2} \text {, função da renda familiar (em múlti- } \\
& \text { plos de salário mínimo); e } \\
& \varepsilon_{\mathrm{jf}}: \text { Termo erro. }
\end{aligned}
$$

Diferentes formas funcionais foram consideradas para representar o efeito da renda sobre a posse de autos, algumas delas buscando diferenciar o padrão de consumo de famílias com diferentes estruturas. A alternativa que produziu melhores resultados e que também apresenta maior simplicidade para análise baseia-se no logaritmo da renda familiar. Esta formulação serviu como base para desenvolvimento do modelo conjunto 1987/1997, que busca também capturar as mudanças dos efeitos dessas variáveis ao longo do tempo.

\subsection{Etapa 3}

As amostras de 1987 e 1997 foram reunidas, tendo sido criada uma variável dummy para representar o ano da pesquisa, adotando-se os valores 0 e 1 para os anos de 1987 e 1997, respectivamente. Preliminarmente à estimação do modelo logit multinomial, foi conduzida nova análise exploratória com o CHAID, para as duas amostras conjuntamente, e para as mesmas variáveis explicativas, além da dummy indicativa do ano da pesquisa. De forma geral, foram reproduzidos os padrões observados na análise realizada na etapa 2, apenas com a amostra de 1987; em particular, verifica-se o efeito não linear do tamanho da família e da idade do chefe, e o impacto negativo do número de trabalhadores quando é controlado o efeito da renda. Um aspecto importante é que a variável indicativa do ano da pesquisa aparece em 
geral após a ocorrência de segmentações com outras variáveis; quando há a segmentação pelo ano, na maioria dos casos registra-se um crescimento da taxa média de autos por família no período. Estes resultados indicam que a especificação obtida com a amostra de 1987 pode ser considerada representativa dos efeitos das variáveis selecionadas sobre a posse de autos familiar.

\subsubsection{Estimação do modelo de posse de autos 1987-1997}

A especificação do modelo de posse de autos 19871997 é baseada na mesma especificação utilizada anteriormente no modelo de posse de autos de 1987, considerando o mesmo conjunto de variáveis, porém para a amostra conjunta das pesquisas de 1987 e 1997. Os efeitos diferenciais ao longo do tempo foram capturados através da inclusão de termos de interação entre cada variável e a dummy indicativa do ano da pesquisa. A especificação adotada produz resultados equivalentes à estimativa de dois modelos independentes, um para cada ano. Porém, a especificação conjunta permite que sejam identificadas diretamente dos resultados aquelas variáveis cujo efeito sofreu alterações significativas ao longo do tempo.

A principal dificuldade refere-se à variável renda familiar. Diversos procedimentos foram utilizados buscando compatibilizar as informações sobre as rendas obtidas em diferentes anos, em um período caracterizado (pelo menos até 1994) por elevados níveis de inflação. Nenhum dos esforços realizados apresentou um resultado satisfatório; ao analisar a distribuição resultante, observa-se uma aparente superposição de duas distribuições diferentes, variando em escalas distintas. Desta forma, a variável renda foi considerada separadamente, associando-se um coeficiente do modelo para valores de 1987 e outro para valores de renda de 1997. Este procedimento dificulta a comparação direta dos coeficientes da renda, mas não prejudica a comparação das elasticidades correspondentes. As funções de utilidade para o modelo logit multinomial de posse de autos familiar para 1987-1997 são dadas por:

$$
\begin{gathered}
\mathrm{U}_{\mathrm{jf}}=\theta_{87 \mathrm{j}} \cdot(1-\mathrm{ANO})+\theta_{97 \mathrm{j}} \cdot \mathrm{ANO}+ \\
+\sum_{\mathrm{i}}\left(\theta_{87 \mathrm{jj}}+\delta_{97 \mathrm{j}} \cdot \mathrm{ANO}\right) \cdot \mathrm{VAR}_{\mathrm{if}}+ \\
+\theta_{\mathrm{REN} 87 \mathrm{j}} \cdot(1-\mathrm{ANO}) \cdot{\mathrm{LOGREN} 87_{\mathrm{jf}}} \\
+\theta_{\mathrm{REN} 97 \mathrm{j}} \cdot \mathrm{ANO} \cdot \mathrm{LOGREN}_{\mathrm{jf}}+\boldsymbol{\varepsilon}_{\mathrm{jf}}
\end{gathered}
$$

em que:

$\mathrm{U}_{\mathrm{jf}}:$ Utilidade associada pela família $\mathrm{f}$ à alternativa $\mathrm{j}$ (número de automóveis: 0, 1, 2, 3 ou mais);

$\theta_{87 \mathrm{j}:}$ Constante específica da alternativa $\mathrm{j}$ para a amostra de famílias de 1987 ;

$\theta_{97 \mathrm{j}}$ : Constante específica da alternativa $\mathrm{j}$ para a amostra de famílias de 1997;

$\theta_{87 \mathrm{ij}}$ : Coeficiente da variável i, específico para a alternativa j, correspondente à amostra de famílias de 1987;

$\delta_{97 \mathrm{ij}}$ : Coeficiente da variável $i$, específico para a alternativa $\mathrm{j}$, correspondente ao efeito diferencial temporal entre os anos de pesquisa;

ANO: Variável dummy ano;

$V A R_{\text {if: }}$ Tamanho da família, (tamanho da família) ${ }^{2}$, número de trabalhadores, número de estudantes, presença ou não de crianças, sexo do chefe, idade do chefe, (idade do chefe) $)^{2}$;

$\theta_{\text {REN 87j }}$ Coeficiente da variável log da renda familiar (LOGREN87) para a amostra de famílias de 1987, específico para a alternativa $\mathrm{j}$;

$\theta_{R E N 97 \mathrm{j}}$ : Coeficiente da variável log da renda familiar (LOGREN97) para a amostra de famílias de 1997, específico para a alternativa $\mathrm{j}$;

$\varepsilon_{\mathrm{jf}}$ : Termo erro.

Pode-se observar que, quando o valor da variável ANO é 0 (para dados referentes à 1987), derivase um modelo específico para 1987, com constante específica própria e coeficientes $\theta_{87}$. Quando ANO igual a 1 (dados de 1997), o modelo correspondente tem constante específica própria e coeficientes das variáveis iguais a $\theta_{87}+\delta_{97}$, com exceção da renda, que tem um coeficiente próprio $\theta_{\mathrm{REN} 97} \mathrm{O}$ valor de $\delta_{97}$ representa o efeito temporal diferencial de cada variável sobre a posse de autos familiar.

\section{RESULTADOS DA ESTIMAÇÃO DO MODELO}

A Tabela 1 mostra as denominações das variáveis utilizadas na estimação do modelo de posse de autos 1987-1997 e a Tabela 2 apresenta as resultados da estimação do modelo logit multinomial de posse de autos 1987-1997. As colunas, identificadas por números $-0,1,2,3+-$ mostram as estimativas dos parâmetros, que representam o efeito de cada uma das variáveis na utilidade de cada alternativa de nível de posse de autos com relação à alternativa "0 
autos". Para a realização da estimação, o LIMDEP atribui valor nulo aos coeficientes da alternativa "0 autos", que é considerada a alternativa-base; isto é necessário uma vez que os valores das variáveis utilizadas - características familiares - são os mesmos para as distintas alternativas de níveis de posse de autos. Os valores sombreados indicam os parâmetros que não foram significativos ao nível de $5 \%$; as estimativas dos parâmetros encontram-se na coluna denominada "coef" e o desvio padrão das estimativas encontra-se na coluna denominada "S".

O LIMDEP fornece uma comparação entre os níveis de posse de autos observados para as famílias da amostra 1987-1997 e aqueles correspondentes à maior probabilidade de escolha, segundo estimada pelo modelo. Foi possível observar que o modelo reproduz razoavelmente bem os dados observados com relação aos níveis de posse de autos do conjunto das famílias, principalmente para a categoria de 0 automóveis, onde se concentra grande parte da amostra (55\% das famílias). Nesta categoria, o modelo superestimou o total de famílias em cerca de $15 \%$; para a categoria 1 auto, o modelo estimou corretamente $92 \%$ das situações familiares, e para as categorias 2 e 3 autos, esse valor é de 51 e $30 \%$ respectivamente. $O$ valor do coeficiente $\rho 2$, que indica a qualidade global do ajuste obtido, situa-se dentro da faixa considerada de boa qualidade.

Tabela 1: Denominação das variáveis independentes no modelo de posse de autos 1987-1997

\begin{tabular}{c|c}
\hline Variável & Denominação \\
\hline Tamanho da família & TAM \\
(Tamanho da família) $^{2}$ & TAM $^{2}$ \\
$\mathrm{~N}^{o}$ trabalhadores na família & TRAB \\
$\mathrm{N}^{0}$ estudantes na família & EST \\
Presença ou não de crianças na família & $\mathrm{CRI}$ \\
Sexo do chefe da família & $\mathrm{SXCH}$ \\
Idade do chefe da família/ 10 & ICH10 \\
(Idade do chefe da família) $)^{2} / 100$ & ICH $^{2} 100$ \\
Log da renda familiar mensal (em múltiplos de salário mínimo) das famílias de 1987 & LOGRE87 \\
Log da renda familiar mensal (em múltiplos de salário mínimo) das famílias de 1997 & LOGRE97 \\
\hline
\end{tabular}

Tabela 2: Estimativa do modelo de posse de autos 1987-1997

\begin{tabular}{|c|c|c|c|c|c|c|c|c|c|}
\hline & & \multicolumn{8}{|c|}{ NÚMERO DE AUTOMÓVEIS } \\
\hline \multirow{2}{*}{\multicolumn{2}{|c|}{ VARIÁVEL }} & \multicolumn{2}{|c|}{0} & \multicolumn{2}{|c|}{1} & \multicolumn{2}{|c|}{2} & \multicolumn{2}{|c|}{$3+$} \\
\hline & & Coef. & $\mathbf{s}$ & Coef. & $\mathbf{s}$ & Coef. & $\mathbf{s}$ & Coef. & $\mathbf{s}$ \\
\hline \multicolumn{2}{|c|}{ CTE87 } & 0 & - & $-16,6849$ & 0,3039 & $-34,9450$ & 0,6331 & $-51,1427$ & 1,4913 \\
\hline \multicolumn{2}{|c|}{ CTE97 } & 0 & - & $-10,2119$ & 0,2395 & $-23,6144$ & 0,4807 & $-34,1555$ & 0,8897 \\
\hline \multirow{2}{*}{ TAM } & 87 & 0 & - & 0,2190 & 0,0484 & 1,0061 & 0,0995 & 1,5618 & 0,2012 \\
\hline & $\delta 97$ & 0 & - & 0,1739 & 0,0697 & 0,1985 & 0,1384 & 0,5618 & 0,2715 \\
\hline \multirow{2}{*}{ TAM $^{2}$} & 87 & 0 & - & $-0,0426$ & 0,0051 & $-0,1305$ & 0,0106 & $-0,1508$ & 0,0197 \\
\hline & $\delta 97$ & 0 & - & $-0,0075$ & 0,0076 & 0,0021 & 0,0153 & $-0,0420$ & 0,0279 \\
\hline \multirow{2}{*}{ TRAB } & 87 & 0 & - & $-0,4097$ & 0,0232 & $-0,4010$ & 0,0393 & $-0,3622$ & 0,0673 \\
\hline & $\delta 97$ & 0 & - & 0,0999 & 0,0356 & 0,0586 & 0,0570 & 0,1287 & 0,0906 \\
\hline \multirow{2}{*}{ EST } & 87 & 0 & - & 0,1130 & 0,0225 & 0,2625 & 0,0405 & 0,0913 & 0,0763 \\
\hline & $\delta 97$ & 0 & - & $-0,1370$ & 0,0336 & $-0,2840$ & 0,0574 & $-0,2252$ & 0,0997 \\
\hline \multirow{2}{*}{ CRI } & 87 & 0 & - & 0,0005 & 0,0470 & $-0,3309$ & 0,0795 & $-0,7840$ & 0,1497 \\
\hline & $\delta 97$ & 0 & - & 0,0497 & 0,0678 & $-0,0701$ & 0,1119 & $-0,7436$ & 0,2082 \\
\hline \multirow{2}{*}{$\mathrm{SXCH}$} & 87 & 0 & - & $-0,8663$ & 0,0501 & $-1,1635$ & 0,1049 & $-1,0066$ & 0,1978 \\
\hline & $\delta 97$ & 0 & - & 0,1074 & 0,0709 & 0,0131 & 0,1448 & $-0,2553$ & 0,2685 \\
\hline \multirow{2}{*}{$\mathrm{ICH} 10$} & 87 & 0 & - & 1,2102 & 0,0776 & 1,9984 & 0,1640 & 3,8577 & 0,4169 \\
\hline & $\delta 97$ & 0 & - & $-0,6294$ & 0,0994 & $-1,1335$ & 0,1972 & $-2,9687$ & 0,4488 \\
\hline \multirow{2}{*}{$\mathrm{ICH}^{2} 100$} & 87 & 0 & - & $-0,1103$ & 0,0080 & $-0,1797$ & 0,0168 & $-0,3337$ & 0,0402 \\
\hline & $\delta 97$ & 0 & - & 0,0545 & 0,0103 & 0,0975 & 0,0202 & 0,2496 & 0,0436 \\
\hline \multicolumn{2}{|c|}{ LOGRE87 } & 0 & - & 1,4224 & 0,0254 & 2,7023 & 0,0450 & 3,4065 & 0,0834 \\
\hline \multicolumn{2}{|c|}{ LOGRE97 } & 0 & - & 1,2395 & 0,0278 & 2,5592 & 0,0481 & 3,3965 & 0,0805 \\
\hline \multicolumn{10}{|c|}{ ESTATÍSTICAS } \\
\hline \multicolumn{2}{|c|}{$\mathrm{N}^{\circ}$ Observações } & \multicolumn{8}{|c|}{41.396} \\
\hline \multicolumn{2}{|c|}{$L(\beta)$} & \multicolumn{8}{|c|}{$-31.241,84$} \\
\hline \multicolumn{2}{|c|}{$L(0)$} & \multicolumn{8}{|c|}{$-41.331,08$} \\
\hline \multicolumn{2}{|c|}{$X^{2}$} & \multicolumn{8}{|c|}{$20.178,47$} \\
\hline \multicolumn{2}{|c|}{ Graus de Liberdade } & \multicolumn{8}{|c|}{57} \\
\hline$\rho^{2}$ & & & & & & & & & \\
\hline
\end{tabular}


A grande maioria dos coeficientes estimados é significativa ao nível de 5\%. Em geral, os coeficientes que não foram significativos estão associados às variáveis que capturam a diferença de comportamento entre 1997 e 1987, indicando que o efeito dessas variáveis não apresentou mudanças ao longo do tempo. Quando a não significância é observada para 1987 (dois casos apenas), indica que a variável em questão não era relevante para definição do nível de motorização familiar naquele ano. Uma dificuldade importante na análise dos resultados deve-se ao fato de que as variáveis utilizadas apresentam em muitos casos correlação elevada, como, por exemplo, entre o número de trabalhadores, ou o número de estudantes, e o tamanho da família.

Os valores das constantes especificas refletem a proporção das diferentes categorias de posse de autos observadas na amostra, sendo crescentemente negativos para níveis mais elevados de posse de autos, tanto para 1987 como para 1997. Como houve um aumento geral da motorização no período, com as famílias migrando para níveis mais elevados de posse de autos, há uma redução do efeito negativo sobre a utilidade de níveis de posse de autos mais elevados, talvez representando condições mais favoráveis em relação aos custos de aquisição e operação dos veículos, que não foram considerados na especificação do modelo.

A análise do efeito do tamanho da família deve levar em consideração conjuntamente os termos de primeiro e segundo grau. Para 1987, ambos os termos são significativos, confirmando o observado anteriormente sobre a forma como esta variável afeta a posse de auto, aumentando inicialmente e decaindo a partir de um valor (uma parábola com o coeficiente do termo quadrático negativo). Em relação aos efeitos temporais, verifica-se que de forma geral esta se tornou uma variável mais importante na determinação dos níveis de posse de auto; o fato que os coeficientes do efeito diferencial nos termos quadráticos não são significativos indica que se manteve, porém, ao longo do tempo, a forma como esta variável exerce sua influência.

O efeito do número de trabalhadores sobre a posse de autos familiar é negativo; as estimativas dos valores dos coeficientes para as três alternativas de posse de autos não são estatisticamente diferentes entre si. Estes resultados confirmam as análises anteriores que indicaram uma redução da taxa média de autos com o aumento do número de trabalhadores na família quando se controla o efeito da renda. Observa-se que, para a categoria mais interessante para análise, a de 1 auto, o efeito diferencial é positivo, indicando a redução da influência negativa associada ao número de trabalhadores durante o período. A relação entre renda e número de trabalha- dores parece passar a afetar menos negativamente a posse de autos.

A importância do número de estudantes mudou entre os anos de pesquisa. Em 1987 o efeito registrado pelo modelo é de aumento da posse de autos com o aumento do número de estudantes. Em 1997 o comportamento é diferente, a presença de um número maior de estudantes não afeta significativamente as decisões familiares relacionadas com a posse de autos, uma vez que o termo diferencial praticamente anula o efeito identificado para 1987.

A presença de crianças na família influencia negativamente a posse de mais de 1 auto, efeito que tendeu a aumentar ao longo do período. Este efeito, observado nas análises anteriores, está correlacionado com o efeito de outras variáveis que descrevem a estrutura familiar, como o tamanho da família, número de estudantes, idade e sexo do chefe da família. A criança em uma família representa um adulto a menos, e possivelmente restringe a atividade remunerada de um dos adultos, refletindo-se sobre a renda.

Os coeficientes da variável sexo do chefe da família indicam que famílias chefiadas por mulheres têm menor probabilidade de possuir autos. $\mathrm{O}$ efeito é semelhante para os diferentes níveis de motorização e não sofreu mudança ao longo do tempo. Este efeito provavelmente resulta de correlações com a renda e com as estruturas de famílias chefiadas por mulheres.

O efeito da variável idade do chefe da família deve levar em conta os termos linear e quadrático que capturam sua influência sobre a posse de autos. Os resultados confirmam o observado nas análises anteriores, com uma elevação da posse de autos até uma certa idade (em geral na faixa de 50 a 60 anos), decrescendo a seguir. Nessa faixa de idade e acima, os filhos mais velhos, que poderiam contribuir para a motorização familiar, em geral já deixaram a casa dos pais. Os efeitos temporais indicam uma redução da importância dessa variável sobre a posse de autos ao longo do tempo. Uma análise gráfica do efeito da idade mostra também que a forma de parábola suavizou-se entre 1987 e 1997, tornando menos importante a variação ligada à idade do chefe da família.

Finalmente, com relação à renda familiar, confirma-se a relevância dessa variável, que contribui positivamente para a motorização familiar, com importância crescente para níveis progressivamente mais elevados de posse de autos. Os coeficientes associados aos valores da renda em 1997 são sistematicamente menores do que os estimados para 1987. Porém, em função dos valores de renda não serem comparáveis nesses dois períodos, foram calculadas as elasticidades médias agregadas (para o 
conjunto da amostra em cada ano) da probabilidade associada a cada nível de posse de autos em relação à renda, assim como para as demais variáveis (Pfeiffer, 2004).

A análise das elasticidades agregadas confirma a redução da importância das variáveis que descrevem a estrutura da família na decisão sobre o seu nível de motorização. A exceção é o tamanho da família, que se torna mais importante, particularmente para as categorias de 1 e 3 ou mais autos. As elasticidades em relação à renda apresentam os valores absolutos mais elevados, confirmando sua importância; porém, entre 1987 e 1997 os valores se reduzem em cerca de $20 \%$ para as categorias de 1 e 3 ou mais autos, sofrendo apenas uma pequena variação positiva para a categoria de 2 autos.

\section{CONCLUSÕES}

O estudo proposto buscou analisar a evolução temporal da posse de autos ocorrida na RMSP entre 1987 e 1997, utilizando um modelo logit multinomial de posse de autos, que considera os fatores sócio-demográficos e econômicos que influenciam o comportamento das famílias, além da captura de efeitos temporais existentes entre os períodos analisados. Os resultados revelaram a existência de uma rede de relações internas à família que afetam as decisões sobre a posse de autos.

De forma geral, ao longo do período considerado, as características familiares mantiveram ou perderam sua importância como fatores explicativos da posse de autos. Este efeito é compatível com os resultados relatados por Strambi et al. (2000), que sugerem uma difusão do automóvel na RMSP nas últimas décadas resultante de sua adoção de forma crescente por sucessivas gerações na população. $\mathrm{O}$ tamanho da família foi uma das únicas variáveis que ganhou importância ao longo do tempo.

A renda, embora um fator importante, também perdeu importância para explicar o nível de motorização familiar. Este resultado é semelhante ao observado na literatura (ver, por exemplo, Dargay e Gately, 1999), que aponta para uma redução da elasticidade da motorização com relação à renda para níveis crescente de renda e de motorização.

$\mathrm{O}$ efeito mais surpreendente observado foi para o número de trabalhadores na família. As expectativas e análises iniciais sugeriam que esta variável fosse correlacionada positivamente com a posse de autos. No entanto, o que se observou foi um efeito contrário - verificado nas análises realizadas com o CHAID e confirmado pelo modelo de posse de autos -, contrapondo-se ao fato de que, na amostra, a taxa média de autos por família aumenta com o número de trabalhadores, até atingir um patamar. $\mathrm{O}$ resultado obtido, porém, é compatível com a hipótese de que, para um dado nível de renda, as famílias com um maior número de trabalhadores contam com mais pessoas exercendo atividades de menor remuneração, com menor poder de consumo individual e familiar.

As conclusões acima revelam que as variáveis de estrutura familiar utilizadas na análise e modelagem da posse de autos perderam importância ao longo do tempo, assim como a própria renda familiar. Isto sugere que as famílias têm passado a dar maior importância a outros fatores para tomar decisões relacionadas com a posse de autos. Alguns desses fatores podem estar vinculados à maior facilidade de aquisição de automóveis por uma família. Facilidades para o financiamento de veículos novos e usados, os consórcios de veículos e a oferta de veículos populares são alguns dos indícios das alternativas disponibilizadas para as famílias que querem adquirir um automóvel. Adicionalmente, a evolução das condições do transporte público, assim como da organização espacial da região podem contribuir para esta tendência.

Agradecimentos

À CAPES e ao CNPq (CT-TRANSPO) pelo apoio à pesquisa. À Secretaria de Transportes Metropolitanos do Estado de São Paulo e à Companhia do Metropolitano de São Paulo pela cessão dos dados das Pesquisas Origem-Destino 1987 e 1997.

\section{REFERÊNCIAS}

Bhart, C. R. e V. A.Pulugurta (1998) Comparison of Two Alternative Behavioral Choice Mechanisms for Households Auto Ownership Decisions. Transportation Research B, v. 32, n. 1, p. 61-75.

Chu, Y. (2002) Automobile Analysis Using Ordered Probit Models. Annual Transportation Research Board Meeting, Washington DC, n. 81, p. 01-31. 1 CD-ROM.

Dargay, J. (2001) The Effect of Income on Car Ownership: Evidence of Asymmetry. Transportation Research A, v. 35, p. $807-821$

Dargay, J. e D. Gately (1999) Income's Effect of Car and Vehicle Ownership, Worldwide: 1960-2015. Transportation Rese$\operatorname{arch} A$, v. 33, n. 2, p. 101-138.

Dargay, J. M. e P. C.Vythoulkas (1999) Estimation of a Dynamic Car Ownership Model: A Pseudo-Panel Approach. Journal of Transport Economics and Policy, n. 33, p. 287-302.

Greene, W. H. (1998) LIMDEP Version 7.0 User's Manual Revised Edition. (1 ${ }^{\mathrm{a}}$ ed.). New York: Econometric Software Inc. 
Jager, J. M. e W. H. Scheltes (1985) Analysis of Automobile Ownership by using a Divise Hierarchical Technique. Transportation Research Record, n. 1037, p. 66-72.

Karlaftis, M. G. e J. Golias (2002) Automobile Ownership, Autoless Households and Urban Traffic Parameters: Are they Related? Annual Transportation Research Board Meeting, Washington DC, n. 81, p. 01-25. 1 CD-ROM.

Kass, G. V. (1980) An Exploratory Technique for Investigating Large Quantities of Categorical Data. Applied Statistics, v. 29, n. 2, p. 119-127.

Kitamura, R. e L. P. Kostyniuk (1986) Maturing Motorization and Household Travel: The Case of Nuclear-Family Households. Transportation Research A, v. 20, n. 3, p. 245-260.

Magidson, J. (1994) The CHAID Approach to Segmentation Modeling: Chi-squared Automatic Interaction Detection. In: Bagozzi, R. P. Advanced Methods of Marketing Research. Cambridge, Mass.

Palma, A. e D. Rochat (2000) Mode Choices for Trips to Work in Geneva: an empirical analysis. Journal of Transport Geography, v. 8, n. 8, p. 43-51.
Prevedouros, P. D. e J. L. Schofer (1992) Factors Affecting Automobile Ownership and Use. Transportation Research Record, n. 1364, p. 152-160.

Pfeiffer, L. M. (2004) Análise e Modelagem da Evolução Temporal da Posse de Autos na Região Metropolitana de São Paulo. 114 p. Dissertação (mestrado) - Escola Politécnica, Universidade de São Paulo.

Purvis, C. L. (1996) Using 1990 Census Public Microdata Sample to Estimate Demographic and Automobile Ownership Models. Transportation Research Record, n. 1443, p. 21-29.

Ryan, J. M. e G. Han (1999) Vehicle-Ownership Model using Family Structure and Accessibility Application to Honolulu, Hawaii. Transportation Research Record, n. 1676, p. 1-10.

Strambi, O; J.-P. Hubert; Y. Bussière e K.-A. van de Bilt (2000) Automobile Patterns of Diffusion in Four Urban Areas: Comparison of Developed and Developing countries. Transportation Research Record, n. 1719, p. 54-60.

Strambi, O e K.-A. van de Bilt (1998) Trip Generation Modeling Using Chaid, a Criterion-based Segmentation Modeling Tool. Transportation Research Record, n. 1645, p. 24-31. 